\title{
Aktuelles aus der Echokardiografie
}

In Wien wurde ein Projekt angedacht, in dessen Rahmen 20 Allgemeinmediziner und Medizinerinnen eine Zusatzausbildung erhalten sollen, die sie „zur

- Durchführung und Beurteilung der Echokardiografie mit 2-dimensionaler Darstellung inkl. TM-Registrierung (inkl. Befunderstellung) sowie

- Farbdopplersonografie des Herzens mit gepulstem und/oder CW-Doppler zur Diagnose, Beurteilung und Kontrolle angeborener oder erworbener Vitien, Beurteilung des pulmonal-arteriellen Druckes und Beurteilung der systolischen und diastolischen Linksventrikelfunktion"-

befähigen und berechtigen soll.

Aus diesem Anlass möchte ich heute einmal über die Ausbildung in Echokardiografie informieren:

Die Ausbildungsrichtlinien für die Durchführung von Ultraschalluntersuchungen, so auch der Echokardiografie wurden über Jahre von den entsprechenden Fachgesellschaften gemeinsam mit der ÖGUM ausgearbeitet und sind in Österreich für die Berechtigung, selbstständig Ultraschalluntersuchungen durchzuführen, verbindend. Darüber hinaus gibt es international akkordierte Qualitätskriterien, wie zum Beispiel klare Richtlinien der ESC, wie eine Zertifizierung zur selbstständigen Durchführung von Echokardiografien zu erlangen ist. Hierzu gibt es auch ein Positionspapier (European Journal of Echocardiografy 2009; 10: 893-905).

Das in Österreich gültige Curriculum für die selbstständige Durchführung von Echokardiografien beinhaltet den Besuch von Grund- und Fortgeschrittenenkursen, deren Inhalt von der AG Echokardiografie der Österreichischen Kardiologischen Gesellschaft festgelegt wurde:

1. Grundkurs (Anatomie, physikalische Grundlagen, Untersuchungstechniken, Normalbefunde, Ventrikelfunkti- on, Klappenerkrankungen [Aortenund Mitralvitien], Perikarderguss, Kardiomyopathien. Insgesamt 36 Stunden, entweder in 2 Wochenenden oder 1 Woche, sollte der Kurs geteilt sein, dann nach Möglichkeit innerhalb eines Abstands von max. 3 Monaten. Ein Drittel der Zeit sollte praktischen Übungen gewidmet sein, wobei 8 Teilnehmer pro Gerät und Tutor die Obergrenze darstellen).

2. Fortgeschrittenenkurs (diastolische Funktion, Endokarditis, kongenitale Vitien, Raumforderungen, Rechtsherzerkrankung, Klappenprothesen, Aortendissektion, Kontrastechokardiografie, Grundlagen der Stressechokardiografie, Indikation zur TEE. Insgesamt 36 Stunden mit praktischen Übungen, entweder in 2 Wochenenden oder 1 Woche; sollte der Kurs geteilt sein, dann nach Möglichkeit innerhalb eines Abstands von max. 3 Monaten).

Danach müssen Fachärzte für Innere Medizin ohne Additivfach Kardiologie und Fachärzte für Kinderheilkunde eine Prüfung in transthorakaler Echokardiografie ablegen, die aus einem theoretischen Teil

- bei FÄ für Innere Medizin in Form eines Multiple-Choice-Tests bzw.

- bei FÄ für Kinder- und Jugendheilkunde in Form einer mündlichen Prüfung bei einem von der Österreichischen Gesellschaft für Kinder- und Jugendheilkunde zu bestimmenden Prüfer und einem praktischem Teil mit Präsentation

- von 300 für FÄ für Innere Medizin bzw.

- von 500 für FÄ für Kinder- und Jugendheilkunde

selbst durchgeführten, auf Video oder einem elektronischen Speichermedium aufgezeichneten echokardiografischen Untersuchungen und Befunden, von denen mindestens

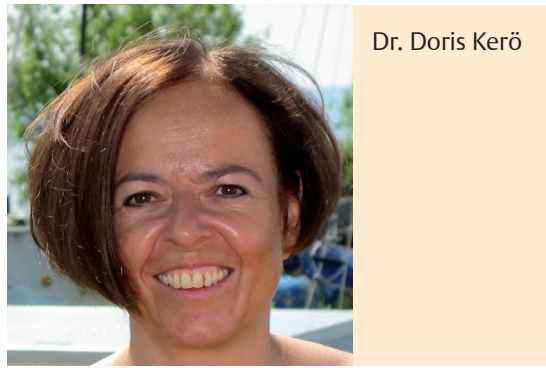

- 50 für FÄ für Innere Medizin bzw.

- 75 für FÄ für Kinder- und Jugendheilkunde

relevante Pathologien aufweisen müssen, besteht. Ergänzt wird die praktische Prüfung durch die selbstständige Untersuchung und Befunderstellung von mindestens 2 Patienten mit relevanten Pathologien in Anwesenheit des Prüfers.

Die ÖGUM ist gerade dabei, gemeinsam mit der DEGUM und SGUM ein Curriculum für Notfallsonografie (für Notfallambulanzen, Notärzte und ggf. auch Allgemeinmediziner) auszuarbeiten mit einer 
fokussierten Echokardiografie als Bestandteil der Ausbildung.

Die Ausbildungsinhalte sind hier:

1. relevante kardiale Anatomie

2. Standard-Ultraschallfenster und Schnittbilder

3. hämodynamische Zusammenhänge

4. Herzfunktion, Klappenfunktion und Perikardergüsse mit und ohne Tamponade

5. linksventrikuläre Funktion (qualitativ)

6. pulmonale Hypertension / Rechtsherzbelastung

7. Zentraqlvenendruck durch Untersuchung der Vena cava inferior

8. dilatierte Aortenwurzel und/oder dilatierte Aorta thoracalis

Die Kursdauer beträgt 8 Stunden, für die Akkreditierung werden 80 Untersuchungen gefordert.

Die kompetente Beurteilung von kompletten Echokardiografien erfordert neben einer gründlichen Ausbildung des Untersuchungsganges selbst ein fundiertes Wissen über die hämodynamischen Gegebenheiten des gesunden wie des kranken Herzens sowie ein ebenso fundiertes Wissen über die anatomischen Gegebenheiten angeborener und erworbener Herzfehler, das man bei Allgemeinmedizinern in keinster Weise einfordern darf. Man kann nicht erwarten, dass ein Allgemeinmediziner, der maximal 12 Monate seiner Ausbildung an einer internen Abteilung verbringt, die nicht zwingend eine kardiologische Abteilung sein muss, dieselben Voraussetzungen im Wissen über Struktur und Hämodynamik des Herzens mitbringt wie ein Kardiologe, dessen spezifische Ausbildung zumindest 8 Jahre dauert. Lediglich eine fokussierte/Basis -Echokardiografie sollte daher auch Inhalt der Echokardiografie für Allgemeinmediziner sein. Darüber hinaus wäre es natürlich wünschenswert, bereits im Medizinstudium echokardiografische und sonografische Grundkenntnisse aller anderen Organe sowie des Bewegungsapparats im Sinne einer „Sonoanatomie“ zu etablieren.

D. Kerö 